\title{
Interference with the lower gut-liver axis induces remission of primary sclerosing cholangitis in a patient with ulcerative colitis
}

\author{
John Ong, ${ }^{1,2}$ Leila Mebarek, ${ }^{2}$ Michael Bath, ${ }^{3}$ Carla Swift, ${ }^{4}$ Babur Javaid, ${ }^{2}$ \\ Jenish Patel, ${ }^{5}$ Yasseen Al-Naeeb ${ }^{2}$
}

\begin{abstract}
To cite: Ong J, Mebarek L, Bath $\mathrm{M}$, et al. Interference with the lower gut-liver axis induces remission of primary sclerosing cholangitis in a patient with ulcerative colitis. BMJ Open Gastro 2018;5:e000239. doi:10.1136/ bmjgast-2018-000239
\end{abstract}

- Additional material is published online only. To view please visit the journal online (http://dx.doi.org/10.1136/ bmjgast-2018-000239).

Received 13 August 2018 Revised 5 October 2018 Accepted 25 0ctober 2018
Check for updates

(C) Author(s) (or their employer(s)) 2018. Re-use permitted under CC BY-NC. No commercial re-use. See rights and permissions. Published by BMJ.

For numbered affiliations see end of article.

Correspondence to Dr John Ong; john.ong@kcl.ac.uk

\section{ABSTRACT}

The gut-liver axis describes the complex interactions between gut microbiota, the small and large bowel, the immune system and the liver. Current evidence associates abnormalities within the gut-liver axis with liver disease such as primary sclerosing cholangitis (PSC). PSC is believed to be an immune-mediated disease though the exact mechanism of its pathogenesis remains unknown. Here, we report a case of a 66 -year-old woman with treatment-resistant ulcerative colitis and PSC which continued to be active following subtotal colectomy. Interestingly, her PSC achieved full remission after proctectomy for treatment-resistant proctitis in the rectal stump. This case report supports existing hypotheses that PSC is an immune-mediated disease triggered by antigens within the gut. More notably, it suggests the yet unidentified pathogens may be localised to the lower gastrointestinal tract including the rectum.

\section{INTRODUCTION}

The gut-liver axis, a concept thought to be first introduced in 1987 by Volta et al, ${ }^{12}$ describes the complex interactions between gut microbiome, the small and large bowel, the immune system and the liver. The gutliver axis plays an important role in the pathogenesis of primary sclerosing cholangitis (PSC). ${ }^{3}$ PSC, an immune-mediated disease, involves chronic inflammation of both intrahepatic and extrahepatic bile ducts, leading to liver cirrhosis. Approximately $47 \%-76 \%$ of patients with PSC also suffer from inflammatory bowel disease (IBD) ${ }^{4}$ though no causal link has been established. Gut microbiome dysbiosis, increased intestinal permeability, translocation of circulating proinflammatory cytokines in the portal vein and circulating autoantibodies are hallmark features in the pathophysiology of PSC $^{3}$, however, little else is known about the triggering microorganism, antigen or its location.
Here, we describe the remission of persistently active PSC in a patient with ulcerative colitis (UC) only after interference of the lower gut-liver axis by means of a two-staged panproctocolectomy. This suggests that the immune-mediated response in PSC is triggered by gut-specific pathogens, and these are localised in the lower gastrointestinal tract (GI) including the rectum.

\section{CASE PRESENTATION}

A 66-year-old woman presented to the surgical outpatient clinic with a 2-month history of bright red rectal bleeding and intermittent diarrhoea. No history of weight loss, foreign travel or dietary change was reported. She suffered from hypertension but had no other comorbidities. She took amlodipine daily and did not drink alcohol or smoke. No significant family history was elicited.

Physical examination was unremarkable, and routine blood tests were normal. A flexible sigmoidosopy however demonstrated mild-moderate inflammation from the rectum to the rectosigmoid junction, and biopsies taken showed features consistent with moderately active UC (see online supplementary file 1). The patient was subsequently started on oral and topical 5-aminosalicylic acids (5-ASAs), but in the following months, her UC worsened and her liver function tests began to rise. Within a year after first presentation, oral steroid therapy in combination with 5-ASAs failed to control the patient's UC, and she was admitted urgently to hospital and referred to the gastroenterology department. However, despite intravenous steroids, antibiotics and ciclosporin rescue therapy, the patient's condition worsened. Her care was transferred back to the 
surgical team, and a subtotal colectomy with loop ileostomy was performed.

The subtotal colectomy was successful, and no complications of the surgery were experienced. However, shortly after discharge from hospital, the patient began to experience right upper quadrant pain and her liver function tests remained persistently raised. At this point, the surgeons referred the patient to a hepatologist for an opinion. PSC was suspected, and a full serological work up for liver disease was performed. This demonstrated antiproteinase 3 antibody (cANCA) positivity only, and the rest of the serological panel was negative. An oesophagogastroduodenoscopy was performed and duodenal biopsies taken, both of which were reported as normal. A magnetic resonance cholangiopancreatography (MRCP) demonstrated a gallstone in the neck of the gallbladder, but no features of cholangiopathy were observed. Given the negative pANCA result, normal biliary tree on MRCP and the patient's reluctance to have a liver biopsy following recent surgery, it was concluded that the cholestatic liver function tests were most likely due to gallstone disease. However, a repeat MRCP to exclude the progression of cholangiopathy was scheduled for 6 months time.

Over the next few months, the patient was referred to a dermatologist after she developed a violet rash near the site of the stoma and over her left lower limb. A punch biopsy was performed on the lesion on her lower limb, and the features of pyoderma gangrenosum were observed, see figure 1 (panel A). Subsequently, the repeat MRCP demonstrated a "double duct sign" (online supplementary file 2), but further CT imaging and endoscopic ultrasound ruled out a pancreatic lesion. Other causes for the double duct sign were not found in our investigations, and IgG4 disease was also excluded (normal IgG4 levels and no IgG4-positive plasma cells seen on biopsies). After discussion at a hepato-pancreatobiliary specialist multidisciplinary meeting, the patient was referred for a liver biopsy as the clinical suspicion for PSC was high, and the patient's liver function tests were persistently elevated. The liver biopsy was performed uneventfully, and features of PSC were noted, see figure 1 (panel B). The patient was treated with ursodeoxycholic acid (UCDA), however, this made little improvement to her cholestatic liver function tests.

Within the same year, the patient was seen in surgical outpatient clinic as a postoperative follow-up. She continued to suffer from ongoing inflammation in the rectum and remnants of the sigmoid colon (rectal stump) despite maximal medical treatment. Importantly, there were no symptoms or clinical evidence that suggested backwash ileitis. As a result, the patient opted for a total proctectomy with ileoanal pouch anastomosis. This was performed once again uneventfully, however, a few months later, the patient represented with symptoms of bowel obstruction. She then proceeded to have a laparotomy for a reversal loop ileostomy and adhesiolysis at site of ileoanal anastomosis. Interestingly, by this point,
(A)

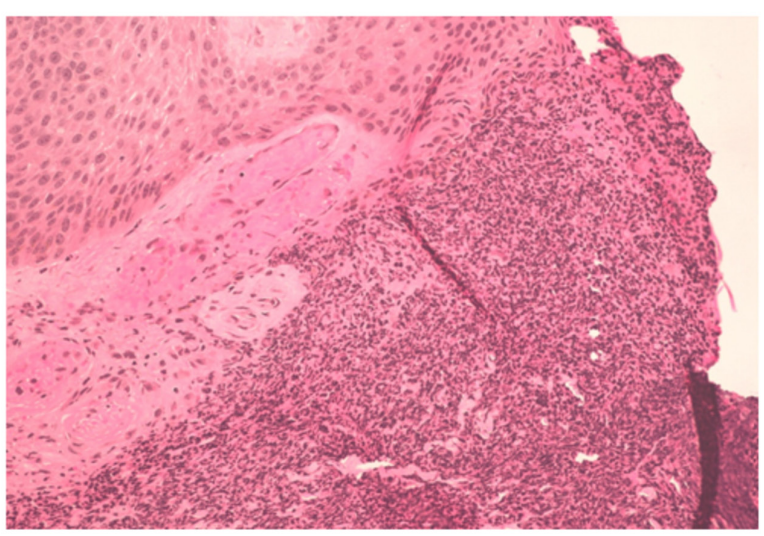

(B)

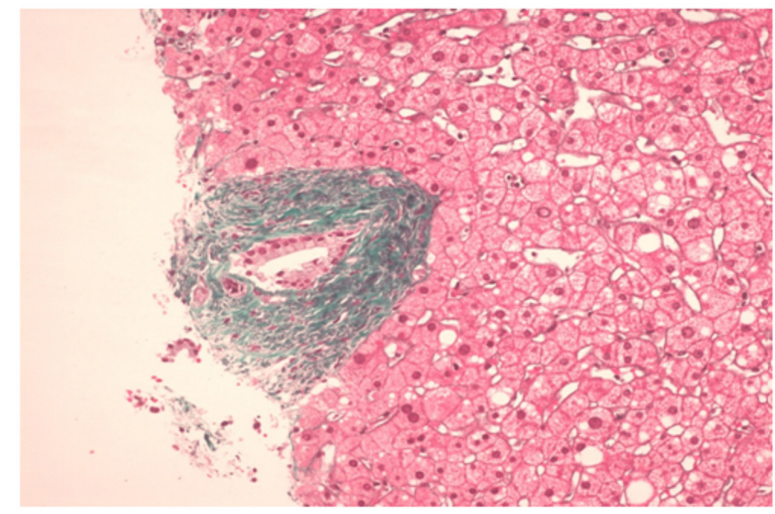

Figure 1 (A) Skin biopsy with diffuse dense neutrophil infiltration confirming diagnosis of pyoderma gangrenosum, H\&E stain at x20 magnification. (B) Liver biopsy demonstrating onion skin appearance of periductal fibrosis highly suggestive for primary sclerosing cholangitis, Masson stain at X20 magnification.

it was noted that the patient's liver function tests had improved significantly (figure 2 ).

The patient was eventually discharged from hospital and she continues to be monitored by the gastroenterology department. Notably, 2 years after her panproctocolectomy her liver function tests normalised. and she has remained asymptomatic. She remains reluctant to have further liver biopsies, but serial imaging of her liver and biliary tree to date have demonstrated complete resolution of the double duct sign and no evidence of chronic liver disease or portal hypertension that would suggest progression of PSC.

\section{DISCUSSION}

The mechanism of pathogenesis in PSC remains poorly understood and this is reflected in the limited treatment options in clinical practice. Current animal models of PSC remain suboptimal, and to date, there is not a single animal model that displays all attributes of the disease seen in humans. ${ }^{5-7}$ Well-designed longitudinal studies offer alternative means to identify causative microorganisms, however, the lack of a biomarker in early disease, 


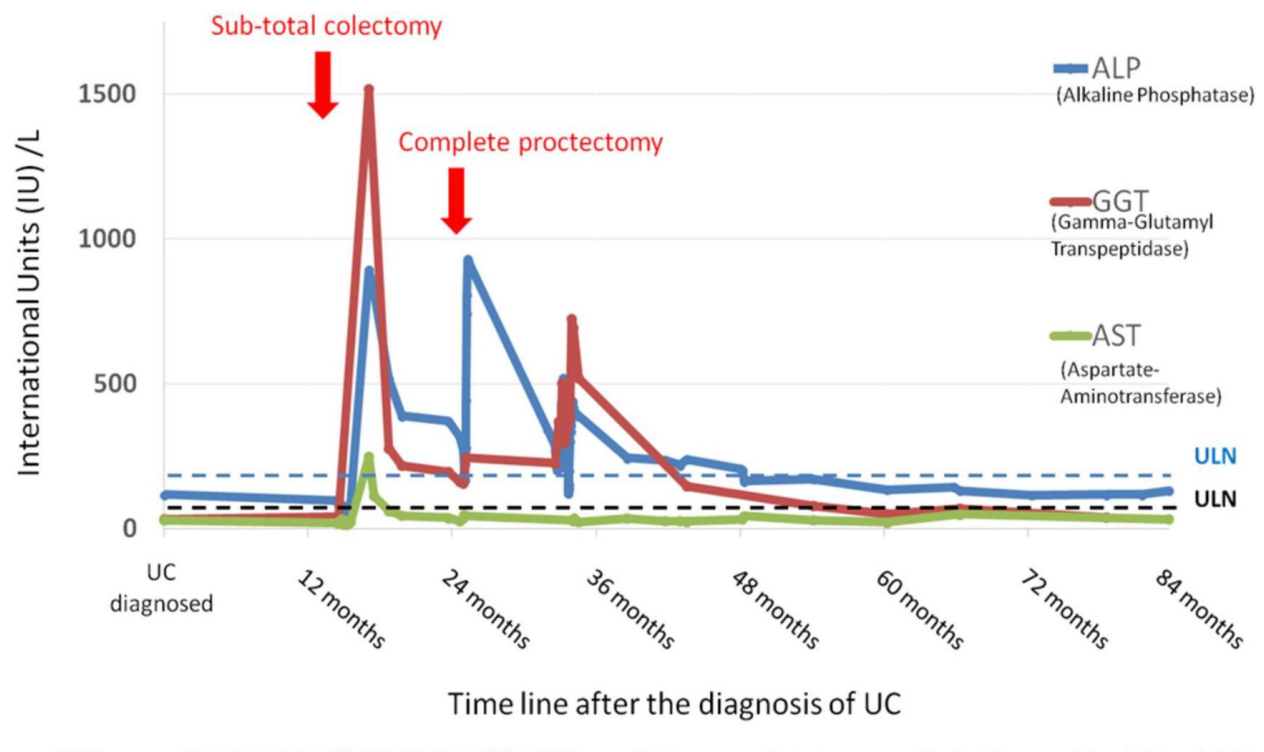

ULN - - - denotes upper limit of normal for ALP ULN - - - denotes upper limit of normal for AST and GGT

Figure 2 The effect of proctocolectomy on liver function tests in primary sclerosing cholangitis. bilirubin is not displayed because the patient never had an abnormal result. UC, ulcerative colitis.

low prevalence of the disease and poor accessibility of the biliary tree restrict the conduct of such studies. ${ }^{5}$ Observational studies such as case control and association studies, though do not demonstrate causality, still provide important information and have contributed to much of our understanding of PSC. ${ }^{3}$

Similarly, this case report describes a series of longitudinal observations in a patient over a 9-year period including the evolution and behaviour of the disease; from the diagnosis of $\mathrm{UC}$, to the development of PSC and pyoderma gangrenosum, and subsequently the effects of partial and complete "knock out" of the lower GI tract by subtotal colectomy and total proctectomy. It is noteworthy that there are several distinct PSC-IBD phenotypes; the majority are patients with PSC-UC ${ }^{7}$ with backwash ileitis, rectal sparing disease or pancolitis. ${ }^{8-10}$ In this particular case, the patient suffered from pancolitis with rectal involvement. It was severe, treatment-resistant pancolitis that was the indication for her colectomy, and there was little clinical evidence to suggest backwash ileitis before or after her surgery. Interestingly, the remission of PSC described in this case is preserved up until today, 7 years following proctectomy. Our observations suggest that the complete remission of PSC was successfully achieved through interference of the lower gut-liver axis, and notably, only after removal of the rectum. This suggests pathogens within the rectum may also contribute to pathogenesis and disease activity of PSC.

Though the pathogenesis of PSC remains poorly understood, the upregulation of toll-like receptors by biliary epithelial cells ${ }^{11}$ and the increase in circulating $\mathrm{T}$ helper 17 cells in patients with PSC $^{12}$ strongly suggest the involvement of the gut microbiome in the pathogenesis of PSC. It has also been proposed that inflammation within the gut causes the propagation of memory $\mathrm{T}$ lymphocytes, and the subsequent recruitment and preferential binding of these chemokine (C-C motif) receptor 9 positive-integrin $\alpha_{4} \beta_{7}$-positive $\mathrm{T}$ lymphocytes to abnormally upregulated mucosal vascular addressin cell adhesion molecule 1, vascular adhesion protein-1 and gut-homing chemokine (C-C motif) ligand (CCL25) on hepatic endothelial cells, cause PSC through inflammation. ${ }^{13}$ This mechanistic model could explain why this patient achieved remission of her PSC after panproctocolectomy, and why association studies have demonstrated a protective effect against the recurrence of PSC in allograft livers in patients with $\mathrm{UC}$ who have had a colectomy. ${ }^{14} 15$

Nonetheless, as the cost of interrogating the microbiome becomes more affordable, longitudinal and high throughput screens of the microbiome in the lower GI tract offers the possibility of identifying the pathogenic microorganisms in PSC and UC. However, even if a pathogen is identified, mechanistic studies to identify a target for drug action and drug safety studies may mean a potential treatment could take decades to transition from bench to bedside. Faecal transplantation may be an alternative method to reverse gut dysbiosis and restore normal balance within the lower GI tract. Interestingly, a clinical trial assessing the effects of faecal transplantation in patients with PSC is already underway, and results of this study are eagerly awaited (ClinicalTrials.gov, identifier: NCT02424175).

In summary, the lower gut-liver axis is most likely involved in the pathogenesis of PSC. Though our understanding of the immune-mediated disease remains poor, rapid advances in basic science research that target the microbiome in the lower GI tract is likely to make headway in developing treatments for the disease.

\section{Author affiliations}

${ }^{1}$ Department of Engineering, University of Cambridge, Cambridge, UK

${ }^{2}$ Department of Gastroenterology, Bedford Hospital South Wing, Bedford, UK 
${ }^{3}$ Department of Surgery, Addenbrooke's Cambridge University Hospital, Cambridge, UK

${ }^{4}$ Department of Gastroenterology, Addenbrooke's Cambridge University Hospital, Cambridge, UK

${ }^{5}$ Department of Histopathology, Bedford Hospital South Wing, Bedford, UK

Acknowledgements $\mathrm{J} 0$ is supported by the W D Armstrong fellowship at Cambridge University.

Contributors J0: wrote the manuscript and performed literature searches. LM, MB: prepared the figures, contributed to parts of the manuscript and performed literature searches. JP: analysed the histological images and contributed to the histology report. YAN, BJ: cared for the patient, obtained consent for images to be used herein and provided the case history. CS: performed literature searches, chased and retrieved investigation results. CS, YAN: edited the manuscript prior to submission.

Funding The authors have not declared a specific grant for this research from any funding agency in the public, commercial or not-for-profit sectors.

Disclaimer The views are expressed by the authors and not necessarily their organisations or the NHS.

Competing interests None declared.

Patient consent Obtained.

Provenance and peer review Not commissioned; externally peer reviewed.

Open access This is an open access article distributed in accordance with the Creative Commons Attribution Non Commercial (CC BY-NC 4.0) license, which permits others to distribute, remix, adapt, build upon this work non-commercially, and license their derivative works on different terms, provided the original work is properly cited, appropriate credit is given, any changes made indicated, and the use is non-commercial. See: http://creativecommons.org/licenses/by-nc/4.0

\section{REFERENCES}

1. Volta U, Bonazzi C, Bianchi FB, et al. IgA antibodies to dietary antigens in liver cirrhosis. Ric Clin Lab 1987;17:235-42.
2. Vairo P, Paolella G, Fasano A. Microbiota and gut-liver axis: their influences on obesity and obesity-related liver disease. J Pediatr Gastroenterol Nutr 2013;56:461-8.

3. Tripathi A. The gut-liver axis and the intersection with the microbiome. Nat Rev Gastroenterol Hepatol 2018.

4. Boonstra K, Beuers U, Ponsioen CY. Epidemiology of primary sclerosing cholangitis and primary biliary cirrhosis: a systematic review. J Hepatol 2012;56:1181-8.

5. Vierling JM. Animal models for primary sclerosing cholangitis. Best Pract Res Clin Gastroenterol 2001;15:591-610.

6. Pollheimer MJ, Trauner M, Fickert P. Will we ever model PSC? - 'it's hard to be a PSC model!' Clin Res Hepatol Gastroenterol 2011;35:792-804.

7. Fickert P, Pollheimer MJ, Beuers U, et al. Characterization of animal models for primary sclerosing cholangitis (PSC). J Hepatol 2014;60:1290-303.

8. Loftus EV, Harewood GC, Loftus CG, et al. PSC-IBD: a unique form of inflammatory bowel disease associated with primary sclerosing cholangitis. Gut 2005;54:91-6.

9. Boonstra K, van Erpecum KJ, van Nieuwkerk KM, et al. Primary sclerosing cholangitis is associated with a distinct phenotype of inflammatory bowel disease. Inflamm Bowel Dis 2012;18:2270-6.

10. Ricciuto A, Kamath BM, Griffiths AM. The IBD and PSC phenotypes of PSC-IBD. Curr Gastroenterol Rep 2018;20:16.

11. Karrar A, Broomé U, Södergren T, et al. Biliary epithelial cell antibodies link adaptive and innate immune responses in primary sclerosing cholangitis. Gastroenterology 2007;132:1504-14.

12. Katt J, Schwinge D, Schoknecht T, et al. Increased T helper type 17 response to pathogen stimulation in patients with primary sclerosing cholangitis. Hepatology 2013;58:1084-93.

13. Eksteen B, Grant AJ, Miles A, et al. Hepatic endothelial CCL25 mediates the recruitment of CCR9+ gut-homing lymphocytes to the liver in primary sclerosing cholangitis. J Exp Med 2004;200:1511-7.

14. Vera A, Moledina S, Gunson B, et al. Risk factors for recurrence of primary sclerosing cholangitis of liver allograft. Lancet 2002;360:1943-4.

15. Alabraba E, Nightingale $P$, Gunson B, et al. A re-evaluation of the risk factors for the recurrence of primary sclerosing cholangitis in liver allografts. Liver Transp/ 2009;15:330-40. 